(2) Open Access Full Text Article

REVIEW

\title{
Innovative fertility preservation strategies and programs for young adults with cancer
}

\author{
This article was published in the following Dove Press journal: \\ Clinical Oncology in Adolescents and Young Adults \\ 27 January 2016 \\ Number of times this article has been viewed
}

\section{Rebecca $\mathrm{H}$ Johnson \\ Division of Pediatric Hematology/ Oncology, Department of Pediatrics, Mary Bridge Hospital, MultiCare Health System, Tacoma, WA, USA}

Correspondence: Rebecca $\mathrm{H}$ Johnson Division of Pediatric Hematology/ Oncology, Department of Pediatrics, Mary Bridge Hospital, MultiCare Health System, PO Box 5299, 3I I South L Street, Tacoma, WA 984I5-0299, USA

$\mathrm{Tel}+$ I 253403348 I

Fax +I 2534034979

Email beckyj100@gmail.com

\begin{abstract}
Preservation of fertility is a key issue for young adults newly diagnosed with cancer. Up to $90 \%$ of cancer patients under the age of 45 are at risk for fertility impairment following cancer therapy. Cancer patients who are not offered fertility preservation (FP) and those who become infertile following therapy may experience long-term psychosocial distress. This review summarizes the numerous effective strategies for preserving fertility, including sperm banking, electroejaculation, and testicular sperm extraction in males and cryopreservation of embryos or oocytes in females. This paper also highlights novel methods currently in development, such as gonadal tissue cryopreservation and in vitro maturation of gametes. In women, anti-Mullerian hormone is emerging as an accurate marker of ovarian reserve, and the use of gonadotropin releasing hormone analogs to protect fertility is increasingly well validated. Although national guidelines mandate FP counseling and referral prior to the start of cancer therapy for patients with reproductive potential, only a minority of young cancer patients in the USA currently take steps to preserve fertility prior to the start of therapy. Some cancer centers across the USA are developing institutional strategies to support FP, resulting in increased utilization of fertility services by newly diagnosed cancer patients.
\end{abstract}

Keywords: young adult, cancer, fertility preservation, program, oocyte, sperm

\section{Cancer in patients of childbearing age}

Active consideration of fertility preservation (FP) options has become a crucial element in the care of all newly diagnosed cancer patients with reproductive potential. ${ }^{1,2}$ Improvements in cancer survival and patient advocacy efforts have led to increased focus on quality of life after cancer treatment, and the potential to have biological offspring is a particularly important element of survivorship. ${ }^{3,4}$

The burden of cancer in people of childbearing age is increasing, due to the increasing incidence of both cancer and second malignant neoplasms in adolescents and young adults (AYAs), decreases in mortality related to therapeutic advances, and delayed childbearing/parenthood. ${ }^{5}$ At present, approximately 150,000 patients with reproductive potential are diagnosed with cancer each year in the USA. ${ }^{6}$ By the age of 40, one in 49 US women is diagnosed with cancer. ${ }^{4}$ Because the incidence of cancer increases with age, ${ }^{7}$ later parenthood means that a cancer diagnosis will overlap with the childbearing years in more individuals. According to data from the Centers for Disease Control and Prevention, the birth rate for US women aged 35-44 years increased by 54\% between 1990 and 2011. More recently, in 2011-2012, birth rates decreased for women in their teens and 20 s but continued to increase for women aged 30 and older. ${ }^{8}$ With this surge in young adult cancer survival and lengthening childbearing years 
for young adults, there is a greater need than ever before to provide FP services for cancer patients.

The FP techniques discussed in this review are clinically available in the USA. Some services described here may not be available in other countries. The cost of FP procedures performed in the operating room is primarily driven by fees of the surgeon, anesthesiologist, and facility. Since charges vary widely by geographic area and institution even within the USA, ${ }^{9}$ costs are cited only when they are published, current, and specific to cancer patients.

\section{Guidelines for counseling newly diagnosed cancer patients about FP}

Because up to $80 \%$ of cancer survivors are at risk for treatment-related infertility, ${ }^{10}$ in recent years, national guidelines in many countries have mandated fertility risk assessment and referral for newly diagnosed cancer patients. These include the American Society of Clinical Oncology (ASCO), who published guidelines in 2006 and updated them in 2013, ${ }^{1}$ the National Comprehensive Cancer Network (NCCN), ${ }^{2}$ the American Society of Reproductive Medicine, the European Society for Medical Oncology, and the European Society of Breast Cancer Specialists. ${ }^{10}$

Per NCCN Clinical Practice Guidelines in Oncology (NCCN Guidelines ${ }^{\circledR}$ ), ${ }^{2}$ providers should discuss FP with all eligible patients prior to start of cancer therapy and consider involving a mental health professional to aid in decisionmaking and facilitate coping with the combined threats of infertility and a new cancer diagnosis. Patients interested in preserving fertility should be referred to a specialist within 24 hours. Importantly, expected prognosis should not be a determinant of which patients should be offered FP. Oncologists are expected to provide each patient with sex-specific counseling regarding his or her risk of infertility resulting from a given cancer therapy, to explain potential treatment options to preserve fertility, and to facilitate referral to a reproductive specialist for expedited treatment.

\section{Barriers to implementation of national FP guidelines}

Prior to the publication of the ASCO fertility guidelines in 2006, low rates of referrals and barriers to effective FP had been described in the literature. ${ }^{11}$ Many perceived barriers related to oncologists' perceptions that there was inadequate time for conversations about FP at the time of cancer diagnosis, discomfort in discussing the topic with young patients, perceived ethical issues regarding conversations with minors, and uncertainty about how to approach the topic of
FP in patients with poor prognosis or developmental delay. Practitioners expressed concern regarding the out-of-pocket cost to patients and the possible resulting inequity of service delivery based on income. Practical barriers to discussions of FP included difficulty finding convenient fertility clinics for referral, as well as urgency to start treatment in patients with unstable clinical status or the potential for rapid clinical deterioration. ${ }^{11}$

Unfortunately, recent studies of FP referral show a striking lack of progress despite the numerous published guidelines above. ${ }^{12,13}$ Only a fraction of qualifying patients currently take steps to preserve fertility prior to the start of cancer treatment. ${ }^{14} \mathrm{~A}$ single institution chart review published in 2012 showed no difference before and after publication of the ASCO FP guidelines in 2006, with fertility consultation offered to only $21 \%$ of eligible patients. ${ }^{15}$ In a 2012 survey of medical oncologists, while $95 \%$ reported routinely discussing fertility risk, a striking $61 \%$ rarely or never referred patients for FP, and 30\% rarely considered a woman's desire for future fertility when considering potential treatment regimens. ${ }^{14}$ In a 2011 survey of pediatric oncologists, the majority acknowledged the importance of FP discussions, but $<50 \%$ referred any patients, and only $12 \%$ referred female patients prior to treatment. ${ }^{16}$

There are significant and ongoing disparities in FP referrals by race, sex, age, and sexual orientation. In a chart review of 231 newly diagnosed cancer patients aged 18-45 years published in 2014, discussion of fertility risk was documented in $26 \%$ of cases, FP options were reviewed in $24 \%$ of cases, and referrals were documented in $13 \%$ of cases. Discussions of fertility risk and/or options were documented significantly less often in Hispanics, females, breast cancer patients, patients aged $\geq 40$ years, and patients with children. ${ }^{17}$ Other studies show provider bias against FP referral for gay men with cancer, as well as low access to FP referral in nonheterosexual women, despite no differences in desire for future pregnancy in this population compared to heterosexual women. ${ }^{6}$

\section{Cancer patients' desire for parenthood}

The majority of cancer survivors rate the ability to have biological offspring as extremely important. In one study of young cancer survivors, $75 \%$ of patients who were childless at diagnosis expressed the desire to have children. ${ }^{18}$ In another study of young survivors aged 18-45 years, most survivors who had wanted children at diagnosis still desired parenthood 3-7 years posttherapy, particularly those who were younger and childless at diagnosis. In addition, $17 \%$ of patients who 
had not wanted children at diagnosis had changed their minds, and later wished for parenthood $(n=55)$. One-third of the young adult cancer survivors surveyed in this study had already experienced problems conceiving, and fertility problems were associated with moderate distress. ${ }^{19}$

\section{Cancer patients' attitudes toward infertility and FP}

Infertility may be unanticipated and may come as a shock to cancer survivors, particularly those who have been inadequately counseled about fertility. ${ }^{20,21}$ Beyond the loss of ability to have children, infertility may be perceived as a loss of masculinity or femininity. ${ }^{22}$

In one study of young male cancer survivors, $80 \%$ felt that sperm banking "helps in the emotional battle against cancer", including those who declined to bank, those who were not successful, and those who banked but never used the stored semen. ${ }^{23}$ FP was seen, even by patients with a poor prognosis, as a positive and future-oriented step that helped them envision life after cancer therapy. ${ }^{23}$ In a survey of men who banked sperm prior to cancer therapy, 96\% said they would recommend sperm banking to other patients, and banking was associated with a sense of psychological relief. ${ }^{24}$ Male cancer survivors who did not bank sperm, and had concerns about infertility, were more likely to report ongoing low self-esteem, conflict in intimate relationships, concern about being rejected, and risky sexual behavior following the completion of cancer therapy. ${ }^{24}$

Due to the relative cost and complexity of FP in women compared to men, fewer women than men take steps to preserve fertility prior to cancer therapy. Female cancer patients report fertility-related distress more frequently than males with cancer. Distress is highest in women who are younger, childless, and non-White. ${ }^{24}$

\section{Assessment of the risk of infertility caused by cancer therapy}

Multiple tools exist for estimating risk to fertility posed by cancer treatment, including the LIVESTRONG fertility risk tool $^{25}$ and Children's Oncology Group long-term follow-up guidelines. ${ }^{26}$ Risk for infertility with cancer therapy has been well described and varies with sex, therapy, dose, and patient age. ${ }^{8,27,28}$ Alkylating agents are particularly gonadotoxic, and there is still limited data on the gonadotoxic effects of newer agents such as tyrosine kinase inhibitors and monoclonal antibodies. ${ }^{29,30}$ Radiation therapy to the brain or gonads poses an independent risk to fertility. Risk for infertility increases with higher doses of chemotherapy, simultaneous use of multiple agents, and radiotherapy in combination with alkylators..$^{8,27}$

Unfortunately, even in the current era, fertility risk assessment calculators present a fairly wide range of possible fertility outcomes for each treatment regimen, and precise assessment of an individual's specific risk is difficult. "Highrisk" regimens lead to $>80 \%$ risk of long-term infertility, intermediate risk protocols confer a $20 \%-80 \%$ risk for infertility, and low-risk treatments are associated with $<20 \%$ incidence of infertility. ${ }^{27}$

\section{Risk for infertility in males after cancer therapy}

Cancer itself has the potential to cause low sperm counts, particularly in patients with Hodgkin lymphoma and testicular cancer. Chemotherapy and/or radiation can cause DNA damage and decrease sperm count and motility. Surgery to reproductive organs such as the testes can impair fertility; pelvic surgery can cause nerve damage and interfere with ejaculation. Factors that determine the likelihood of reproductive damage include drug type and dosage, radiation location and dosage, pubertal status at the time of treatment, and pretreatment fertility, which is often unknown. ${ }^{31,32}$

Azoospermia is common on completion of therapy and may persist for variable periods of time. ${ }^{27}$ Natural recovery of sperm production has been reported to occur as late as 9 years after the completion of cancer therapy. ${ }^{33}$ The presence of adequate virilization in male survivors is not an accurate predictor of current or future fertility. Leydig cells, which produce testosterone, are much more resistant to injury from gonadotoxic chemotherapy and radiation than germ cells. ${ }^{34}$ Leydig cell dysfunction occurs most commonly in cancer patients who have received combination chemoradiotherapy, but it may also occur after high-dose chemotherapy without total body irradiation. Patients may appear well-virilized, and yet be infertile. ${ }^{35}$

\section{Risk for infertility in females after cancer therapy}

In contrast to males, in whom infertility is common immediately following therapy but may resolve over time, females are at risk for irreversible premature ovarian failure, which may occur many years after the completion of cancer therapy. ${ }^{27}$ Thus, even if menses and ovulation appear normal following therapy, young female cancer survivors are at risk for a shortened reproductive window compared to the general population. ${ }^{36,37}$ 
Chemotherapy and radiation therapy can damage the primordial follicles in the ovary and deplete ovarian reserve. The degree of damage depends on drug, dose, and age of the patient. Older female AYAs in their 30s are more likely than younger women to undergo premature menopause following chemotherapy, since older women's stores of primordial follicles are not as large. ${ }^{36}$ Women may resume menstrual cycling after the completion of cancer therapy, yet still be at risk for subsequent premature ovarian failure due to a diminished store of primordial follicles. In one study of young women who received both alkylating chemotherapy and radiotherapy before the age of $20,42 \%$ had reached menopause by 31 years of age, compared to $5 \%$ of healthy controls. $^{36}$

\section{FP options for males}

Sperm banking is the established, gold standard method of preserving fertility in postpubertal males., ${ }^{3,27}$ Compared to the available methods for female FP, this technique is relatively simple, rapid, and affordable. In the USA, sperm banking costs approximately $\$ 1,000$ upfront, followed by storage fees of \$300-\$500 annually. ${ }^{38}$ The best yield is obtained when men bank sperm prior to the start of gonadotoxic chemotherapy ${ }^{38}$ and when they provide a fresh semen sample to a sperm bank. For men who live in remote locations with no nearby sperm bank, mail-in kits are also available. ${ }^{3}$ According to ASCO guidelines, ${ }^{1}$ sperm banking should be offered to all men who have reproductive potential. The population of men most likely to choose sperm banking are those who are younger, more highly educated, and/or childless. ${ }^{24}$ Sperm banking is preferred over any other method of FP for all men who have the physical capacity to masturbate. ${ }^{3,32}$

Electroejaculation is a method of obtaining a semen sample by vibratory stimulation of the penis. This method is safe and well studied. ${ }^{32,39}$ It is a noninvasive option for patients with physical or cultural barriers to masturbation and for patients who are unsuccessful in attempts to bank sperm. ${ }^{3,32}$ In a series of adolescent boys who had failed masturbation, electroejaculation yielded adequate semen samples for cryopreservation in $45 \%$ of cases. ${ }^{40}$

Testicular sperm extraction is a procedure in which semen is extracted from the seminiferous tubules by percutaneous needle aspiration. This strategy may benefit several populations of patients, including early pubertal males and men with physical barriers to masturbation, such as those with paralysis or sensory changes related to a spinal cord tumor. ${ }^{32,39}$ This procedure can also be used, 6 or more months after the completion of chemotherapy, to obtain sperm for in vitro fertilization (IVF) and intracytoplasmic sperm injection in male cancer survivors with azoospermia. ${ }^{3}$

Sperm should be banked before the start of therapy if at all possible. ${ }^{1}$ A period of testicular dysfunction is common following even "low-risk" chemotherapy protocols, and ongoing chemotherapy impairs spermatogenesis. One study analyzed men with relapsed leukemia who attempted to bank sperm after initial therapy and prior to bone marrow transplant. The majority of patients had impaired sperm production; azoospermia was present in 39\% of patients and oligospermia in $15 \%{ }^{38}$

\section{FP options for females}

Due to its relatively higher cost, complexity, and potential to delay therapy in women, FP is utilized less frequently by females than by males. ${ }^{24,41}$ Recent studies indicate that FP is increasing but still used by only a minority $(2 \%-12 \%)$ of women at risk for cancer-related infertility. However, an additional 19\% of young women in the study altered their cancer treatment plan based on fertility considerations, choosing one chemotherapy regimen over another or refusing endocrine therapy. ${ }^{24,42}$

\section{Surgical options to protect ovaries from radiation damage}

When possible, cancer surgery can be planned to spare the reproductive organs, preserving the potential for fertility. Ovarian transposition/oophoropexy is commonly used prior to radiation therapy in the pelvis. Ovaries are moved out of the radiation field. ${ }^{27}$ This procedure can reduce the radiation dose to the ovaries by up to $95 \%$. Following transposition, $60 \%-89 \%$ survivors under 40 years of age maintain ovarian function, although some studies suggest that only $15 \%$ may be fertile. Natural conception is possible in some cases, but IVF may be required for conception if the ovary has been moved out of proximity from the fallopian tube. ${ }^{24}$

\section{Cryopreservation of embryos or oocytes for FP}

For decades, embryo cryopreservation has been the traditional gold standard method of preserving fertility in women. This technique requires sperm from a partner or donor to fertilize the oocytes prior to freezing. Thus, it is a viable option for women who are stably partnered, but it may be a less attractive option for young women who have not yet chosen a life partner. ${ }^{3}$

Oocyte cryopreservation is a technique in which drugs are given to stimulate ovulation, after which oocytes are harvested and frozen for later fertilization. The process has 
become increasingly well established; over 1,000 live births have been documented. ${ }^{9}$ In 2012, American Society of Reproductive Medicine stated that oocyte cryopreservation was no longer experimental and should be considered standard of care. ${ }^{8}$ The technique has good yield for cancer survivors: in many studies, the number of oocytes frozen following stimulation is equivalent for cancer survivors and age-matched infertile controls without cancer. ${ }^{43-45}$ Oocyte retrieval rates vary according to diagnosis, with higher retrieval rates in women with breast and gynecological cancers compared to those with hematologic malignancies. ${ }^{46,47}$

Ovarian stimulation protocols take 10-14 days to complete and can be started at any time of the menstrual cycle. ${ }^{8}$ Thus, oocyte cryopreservation prior to the start of therapy is an option only for patients who can safely delay the initiation of chemotherapy by 2 weeks. ${ }^{3}$ For women with adequate ovarian reserve following cancer treatment, the procedure may also be attempted 6 or more months following the completion of therapy. ${ }^{8}$

\section{Efficacy of embryo vs oocyte cryopreservation}

Despite initial concerns that oocyte cryopreservation would be less effective than embryo cryopreservation, multiple studies have documented equal success with oocyte and embryo cryopreservation. The live birth rate and risk of embryonic aneuploidy for both methods is equivalent to that of IVF using fresh oocytes. ${ }^{48}$ Clinical estimates of success for any given woman must take into account her age, ovarian reserve, and previous treatment status. Cost is comparable: oocyte cryopreservation costs approximately $\$ 11,900$ and embryo freezing, approximately $\$ 12,400$ per cycle. $^{8}$

\section{Emerging methods for FP in females \\ Ovarian tissue cryopreservation \\ and transplantation}

Ovarian tissue cryopreservation is available in the context of clinical trials at some US cancer centers. ${ }^{3}$ Slices of ovarian cortex are retrieved in a laparoscopic procedure prior to the start of cancer therapy, frozen, and later thawed and laparoscopically grafted onto the patient's ovary, the pelvic wall or abdominal wall. The first live births using this technique were documented in the 1950s in animal models and in 2004 in a human cancer survivor. Since then, more than 37 children have been born following ovarian tissue cryopreservation. After the transplantation procedure, hormonal function returns to normal and fertility is restored in many cancer survivors. Natural conception, without the aid of IVF, is often possible when the cryopreserved tissue is grafted onto an ovary and near a fallopian tube. ${ }^{49}$

Potential risks of ovarian tissue cryopreservation and transplantation include loss of follicles in the transplanted tissue due to ischemia, as well as the risk of transferring malignant cells harbored in the gonad at the time of freezing. ${ }^{3}$ Disease recurrence has been reported in a patient with leukemia. ${ }^{50}$ Malignant cells in the ovary have been documented in $8 \%-55 \%$ of women who died of a various types of cancer under 40 years of age. ${ }^{51}$ In one study of female leukemia patients with no leukemic involvement of the ovaries by histologic examination; malignant cells were detectable in the ovaries by polymerase chain reaction in 30\%-70\% of cases. ${ }^{52}$ Thus, the procedure is contraindicated in the setting of either leukemia or ovarian cancer. ${ }^{53}$

For eligible patients, the technique has several advantages. Although surgery is required, cancer therapy can be initiated immediately thereafter, leading to less delay than techniques that require drugs to stimulate ovulation. A large number of gametes can be obtained in a single procedure. ${ }^{49}$ In addition, ovarian tissue cryopreservation is the only FP option currently available to prepubertal patients. ${ }^{53}$ In contrast to oocyte and embryo cryopreservation, IVF is not required for most conceptions following ovarian tissue reimplantation. ${ }^{49}$

\section{In vitro maturation of primordial follicles into fertilizable oocytes}

A longer-range experimental prospect for FP is the in vitro maturation of immature oocytes harvested from ovarian tissue into oocytes that can be frozen for later use. Primordial follicles are collected in a surgical procedure, matured in vitro, and cryopreserved. The procedure can be performed in combination with ovarian cortex harvesting and cryopreservation. ${ }^{54}$ At least one human live birth has been reported following in vitro maturation of cryopreserved oocytes. ${ }^{55}$ This approach would facilitate FP for prepubertal girls and postpubertal women who cannot delay treatment for oocyte or embryo banking. In contrast to ovarian tissue freezing, this technique would eliminate the need to reimplant tissue, thereby minimizing the risk for tumor contamination. ${ }^{53}$

Several other groundbreaking methods for FP are currently in development, including prepubertal ovarian and testicular tissue cryopreservation, ${ }^{3,56,57}$ as well as living or cadaveric uterine transplants for patients who required hysterectomy as part of their cancer therapy. The first live birth following uterine transplant was recorded in $2014 .{ }^{58}$ In 
addition, the capacity to create pluripotent stem cells from autosomal cells is currently under investigation in animal models. ${ }^{59}$

\section{Protecting ovarian reserve using gonadotropin releasing hormone analogs}

Data are accruing on the use of gonadotropin releasing hormone $(\mathrm{GnRH})$ analogs during cancer therapy to preserve future fertility. These agents create a hormonal state similar to that in prepubertal girls, reducing perfusion to the ovary and limiting direct cytotoxic injury. ${ }^{8}$ Despite study limitations, the majority of trials on the efficacy of GnRH analogs demonstrate benefit: 20 studies, including five randomized controlled trials on 1,837 patients showing decrease in premature ovarian failure with the use of GnRH analogs; nine reports (593 patients) do not support their use. However, all of these latter studies used only postchemotherapy amenorrhea as an endpoint, which is not an accurate predictor of subsequent fertility. ${ }^{4}$

Use of GnRH agonists is further supported by seven meta-analyses including Cochrane's, which concluded that these agents preserve menstrual function in $91 \%$ of treated survivors vs $41 \%$ of controls. Importantly, increased fecundity was also noted: pregnancy occurred in $19 \%-71 \%$ of treated survivors (odds ratio $[\mathrm{OR}]=12.87 ; P=0.001$ ). ${ }^{4}$ The recent Prevention of Early Menopause Study (POEMS, SWOG S0230), a Phase III randomized breast cancer trial, showed that the leuteinizing hormone-releasing hormone analog goserelin given along with chemotherapy was associated with less premature ovarian failure ( $\mathrm{OR}=0.3, P=0.04)$, more pregnancies $(21 \%$ vs $11 \%)(P=0.03)$, and, interestingly higher disease-free (hazard ratio [HR] 0.49, $P=0.04$ ) and overall survival (HR 0.43, $P=0.05$ ). ${ }^{60}$

GnRH-analogs are regarded as safe for use in women with all types of cancer. Two years of therapy with goserelin was reported to cost $\$ 5,532$ in one study. ${ }^{61} \mathrm{GnRH}$ analogs can be used to prevent menorrhagia in the setting of thrombocytopenia. ASCO guidelines suggest that they be used in parallel with chemotherapy and in addition to other FP strategies. ${ }^{4}$

\section{Measuring ovarian reserve: anti-Mullerian hormone}

Serum anti-Mullerian hormone (AMH) level is emerging as an accurate predictor of ovarian reserve and risk of premature ovarian failure, which will aid clinical decision-making regarding fertility interventions before and after cancer therapy. AMH is a hormone secreted by primordial follicles in the ovary. AMH levels do not fluctuate significantly through the menstrual cycle, and AMH is undetectable following menopause or bilateral oophorectomy. ${ }^{62}$ AMH levels are generally normal in premenopausal breast cancer patients at the time of diagnosis, but are often decreased in women with newly diagnosed hematologic malignancies. Higher AMH levels prior to therapy predict high posttreatment levels, and presumably higher fecundity. For reasons that are not well-understood, low AMH levels correlate more strongly with decreased fecundity in older premenopausal women compared to younger women. ${ }^{62}$

AMH levels decrease drastically following chemotherapy, suggesting acute injury to primordial follicles. ${ }^{62}$ More intensive chemotherapy regimens ${ }^{63}$ and regimens including alkylating agents ${ }^{64}$ are associated with more delayed recovery of AMH following the completion of therapy. Consistent with previous observations that AYA women in their 30s are more likely than younger women to develop chemotherapy-associated premature ovarian failure, AMH recovery following chemotherapy is more likely in younger, compared to older AYAs with a history of Hodgkin's lymphoma. ${ }^{65}$

\section{Innovations among US FP programs}

Following the publication of the ASCO FP guidelines in $2006,{ }^{66}$ a number of US cancer centers developed formal institutional mechanisms to support FP in newly diagnosed cancer patients with reproductive potential.

\section{Fertile Hope Centers of Excellence}

In 2005, the not-for-profit organization Fertile Hope, which was later acquired by LIVESTRONG, developed a center of excellence program to recognize outstanding FP practices at cancer centers ${ }^{67}$ For inclusion as a center of excellence, institutions were required to demonstrate institutional commitment for FP provision of professional education on FP, dissemination of patient education materials, creation of institutional systems to notify patients about infertility risks at diagnosis, and an efficient process for FP referrals. Nine institutions achieved center of excellence status during the 7-year initiative. Automated electronic systems to identify new patients were noted to be extremely effective in facilitating FP. ${ }^{67}$ Many elements from the Fertile Hope strategy have been incorporated into current FP programs throughout the USA..$^{6,68,69}$

\section{Optimizing institutional standard practices}

Developing institutional best practices to support FP can be instrumental in the creation of FP practices that follow the ASCO guidelines. ${ }^{1}$ Over the past decade, several institutions 
within the USA have documented increased FP in newly diagnosed cancer patients. ${ }^{69-71}$ Between different institutions, strategies to accomplish this outcome shared some similarities and contained other novel elements. At the Moffitt Cancer Center, simply providing educational brochures to newly diagnosed cancer patients of childbearing age raised the number of patient calls to the partner reproductive endocrinology center by ninefold, to a high of $27 \% .{ }^{70}$ At Seattle Children's Hospital, the implementation of a standard process for offering FP in 2009 resulted in increased rates of sperm banking attempts from $8 \%$ to $70 \%$ of eligible postpubertal AYA males with cancer. ${ }^{69}$ Development of a similar system at Northwestern University, aimed at young adult males, increased the rate of fertility consultations for sperm banking from $23 \%$ to $43 \%{ }^{71}$

Creation of institutional policies to support FP can be facilitated by practices such as holding a stakeholders' workshop to solidify collaborations with regional fertility clinics and to define staff responsibilities, steps for implementation, and metrics to track. ${ }^{69}$ The designation of a navigator with dedicated time for FP may streamline the process and offload responsibilities from the attending oncologist. ${ }^{6,69}$

\section{Multidisciplinary teams}

Some US cancer centers have promoted FP by incorporating fertility counseling into the work of the multidisciplinary care team. Moffitt Cancer Center formally included genetic counselors and reproductive specialists into its multidisciplinary care team for young breast cancer patients. The team also includes surgeons, medical oncologists, radiation oncologists, radiologists, and social workers. ${ }^{68}$ The University of North Carolina employs a multidisciplinary strategy that also includes pathologists and laboratory personnel among its team members to help facilitate tissue banking. ${ }^{6}$

The development of efficient and standardized systems to support FP within institutions has the potential to improve both psychosocial outcomes and rates of FP in AYAs with cancer. ${ }^{21,69-72}$ Cancer patients who did not receive fertility counseling report low self-esteem, regret for not proactively questioning their infertility risks, guilt for not being able to conceive a child for their partner, and/or fear of never finding a partner. ${ }^{73,74}$ Women who have received fertility counseling report less regret and better quality of life than those who did not. ${ }^{21}$

\section{Conclusion}

Cancer-related infertility can cause long-term distress and impair quality of life in survivors. ${ }^{75}$ Despite the mandates by $\mathrm{ASCO}^{1}$ and other guidelines that $\mathrm{FP}$ be routinely offered, a study published in 2013 indicated that FP services are often not integrated into the care provided by National Cancer Institute-designated comprehensive cancer centers. Onethird of programs had neither on-site fertility clinics nor off-site partners offering FP services. Two-thirds of programs did provide FP services on-site or through partner institutions, but institutional commitment for the service varied; only $27 \%$ of all programs surveyed had staff with dedicated time for patient counseling, and only $13 \%$ had institutional policies regarding consistent provision of information. ${ }^{76}$ However, recent statistics indicate an uptick in the number of patients utilizing FP prior to cancer therapy. ${ }^{24,77}$ With the trend toward delayed parenthood in the USA, ${ }^{8}$ increase in cancer incidence with age, ${ }^{7}$ and escalating incidence of several AYA cancers, the need for FP is expected to continue to increase in coming years. ${ }^{5}$ Fortunately, the number of FP choices available to young adults with cancer also continues to grow.

\section{Disclosure}

The author is a member of the Speaker's bureaus of Baxalta and Jazz Pharmaceuticals. The author reports no other conflicts of interest in this work.

\section{References}

1. Loren AW, Mangu PB, Beck LN, et al; American Society of Clinical Oncology. Fertility preservation for patients with cancer: American Society of Clinical Oncology clinical practice guideline update. J Clin Oncol. 2013;31(19):2500-2510.

2. Coccia PF, Pappo AS, Altman J, et al. Adolescent and young adult oncology, version 2.2014. J Natl Compr Canc Netw. 2014;12(1):21-32; quiz 32.

3. Johnson RH, Kroon L. Optimizing fertility preservation practices for adolescent and young adult cancer patients. J Natl Compr Canc Netw. 2013;11(1):71-77.

4. Blumenfeld Z, Katz G, Evron A. 'An ounce of prevention is worth a pound of cure': the case for and against GnRH-agonist for fertility preservation. Ann Oncol. 2014;25(9):1719-1728.

5. Go RS, Gundrum JD. Cancer in the adolescent and young adult (AYA) population in the United States: current statistics and projections. J Clin Oncol. 2011;29(Suppl). ASCO Annual Meeting 2011, abstract 6072.

6. Kim J, Kim KH, Mersereau JE. Building a successful fertility preservation program at a major cancer center. $J$ Gynecol Oncol. 2014; 5(2):148-154.

7. Bleyer A. Young adult oncology: the patients and their survival challenges. CA Cancer J Clin. 2007;57(4):242-255.

8. Ross L, Chung K, Macdonald H. Fertility preservation in the female cancer patient. J Surg Oncol. 2014;110(8):907-911.

9. Lee R, Li PS, Goldstein M, Tanrikut C, Schattman G, Schlegel PN. A decision analysis of treatments for obstructive azoospermia. Hum Reprod. 2008;23(9):2043-2049.

10. Linkeviciute A, Boniolo G, Chiavari L, Peccatori FA. Fertility preservation in cancer patients: the global framework. Cancer Treat Rev. 2014;40(8):1019-1027.

11. Schover LR, Brey K, Lichtin A, Lipshultz LI, Jeha S. Oncologists' attitudes and practices regarding banking sperm before cancer treatment J Clin Oncol. 2002;20(7):1890-1897. 
12. Adams E, Hill E, Watson E. Fertility preservation in cancer survivors: a national survey of oncologists' current knowledge, practice and attitudes. Br J Cancer. 2013;108(8):1602-1615.

13. Kumar A, Merali A, Pond GR, Zbuk K. Fertility risk discussions in young patients diagnosed with colorectal cancer. Curr Oncol. 2012; 19(3):155-159.

14. Forman EJ, Anders CK, Behera MA. A nationwide survey of oncologists regarding treatment-related infertility and fertility preservation in female cancer patients. Fertil Steril. 2010;94(5):1652-1656.

15. Goodman LR, Balthazar U, Kim J, Mersereau JE. Trends of socioeconomic disparities in referral patterns for fertility preservation consultation. Hum Reprod. 2012;27(7):2076-2081.

16. Köhler TS, Kondapalli LA, Shah A, Chan S, Woodruff TK, Brannigan RE. Results from the survey for preservation of adolescent reproduction (SPARE) study: gender disparity in delivery of fertility preservation message to adolescents with cancer. JAssist Reprod Genet. 2011;28(3):269-277.

17. Quinn GP, Block RG, Clayman ML, et al. If you did not document it, it did not happen: rates of documentation of discussion of infertility risk in adolescent and young adult oncology patients' medical records. J Oncol Pract. 2015;11(2):137-144.

18. Schover LR. Patient attitudes toward fertility preservation. Pediatr Blood Cancer. 2009;53(2):281-284.

19. Armuand GM, Wettergren L, Rodriguez-Wallberg KA, Lampic C. Desire for children, difficulties achieving a pregnancy, and infertility distress 3 to 7 years after cancer diagnosis. Support Care Cancer. 2014; 22(10):2805-2812.

20. Mulhall JP, Applegarth LD, Oates RD, Schlegel PN, editors. Fertility Preservation in Male Cancer Patients. Cambridge, UK: Cambridge University Press; 2013.

21. Letourneau JM, Ebbel EE, Katz PP, et al. Pretreatment fertility counseling and fertility preservation improve quality of life in reproductive age women with cancer. Cancer. 2012;118(6):1710-1717.

22. Ussher JM, Perz J, Gilbert E; Australian Cancer and Sexuality Study Team. Perceived causes and consequences of sexual changes after cancer for women and men: a mixed method study. BMC Cancer. 2015; $15: 268-286$

23. Crawshaw MA, Glaser AW, Hale JP, Sloper P. Young males' experiences of sperm banking following a cancer diagnosis - a qualitative study. Hum Fertil (Camb). 2008;11(4):238-245.

24. Brezina PR, Kutteh WH, Bailey AP, Ding J, Ke RW, Klosky JL. Fertility preservation in the age of assisted reproductive technologies. Obstet Gynecol Clin North Am. 2015;42(1):39-54.

25. LIVESTRONG fertility risk tool [homepage on the Internet]. Available from: http://www.livestrong.org/we-can-help/fertility-services/risks/. Accessed May 28, 2015.

26. Children's Oncology Group. Long-Term Follow-Up Guidelines for Survivors of Childhood, Adolescent, and Young Adult Cancer (2013) [homepage on the Internet]. Available from: http://www.survivorshipguidelines. org/pdf/LTFUGuidelines_40.pdf. Accessed February 20, 2015.

27. Levine J, Canada A, Stern CJ. Fertility preservation in adolescents and young adults with cancer. J Clin Oncol. 2010;28(32):4831-4841.

28. Kenney LB, Cohen LE, Shnorhavorian M, et al. Male reproductive health after childhood, adolescent, and young adult cancers: a report from the Children's Oncology Group. J Clin Oncol. 2012;30(27):3408-3416.

29. Bhandari A, Rolen K, Shah BK. Management of chronic myelogenous leukemia in pregnancy. Anticancer Res. 2015;35(1):1-11.

30. Lambertini M, Peccatori FA, Azim HA Jr. Targeted agents for cancer treatment during pregnancy. Cancer Treat Rev. 2015;41(4): 301-309.

31. Fertile Hope. Cancer and fertility: fast facts for professionals [homepage on the Internet]. New York, NY: Fertile Hope; 2007. Available from: http:/www.ohsu.edu/xd/health/services/cancer/getting-treatment/ why-ohsu/for-healthcare-professionals/continuing-ed/upload/Cancerand-Fertility-for-HCP.pdf. Accessed February 20, 2015.

32. Tournaye H, Dohle GR, Barratt CL. Fertility preservation in men with cancer. Lancet. 2014;384(9950):1295-1301.
33. Anserini P, Chiodi S, Spinelli S, et al. Semen analysis following allogeneic bone marrow transplantation. Additional data for evidence-based counselling. Bone Marrow Transplant. 2002;30(7):447-451.

34. Brydøy M, Fosså SD, Dahl O, Bjøro T. Gonadal dysfunction and fertility problems in cancer survivors. Acta Oncol. 2007;46(4):480-489.

35. Somali M, Mpatakoias V, Avramides A, et al. Function of the hypothalamic-pituitary-gonadal axis in long-term survivors of hematopoietic stem cell transplantation for hematological diseases. Gynecol Endocrinol. 2005;21(1):18-26.

36. Brougham MF, Wallace WH. Subfertility in children and young people treated for solid and haematological malignancies. Br J Haematol. 2005;131(2):143-155.

37. Green DM, Sklar CA, Boice JD Jr, et al. Ovarian failure and reproductive outcomes after childhood cancer treatment: results from the Childhood Cancer Survivor Study. J Clin Oncol. 2009;27(14):2374-2381.

38. Nahata L, Cohen LE, Lehmann LE, Yu RN. Semen analysis in adolescent cancer patients prior to bone marrow transplantation: when is it too late for fertility preservation? Pediatr Blood Cancer. 2013; 60(1):129-132.

39. Nangia AK, Krieg SA, Kim SS. Clinical guidelines for sperm cryopreservation in cancer patients. Fertil Steril. 2013;100(5):1203-1209.

40. Adank MC, van Dorp W, Smit M, et al. Electroejaculation as a method of fertility preservation in boys diagnosed with cancer: a single-center experience and review of the literature. Fertil Steril. 2014;102(1): 199-205.

41. Williams DH. Sperm banking and the cancer patient. Ther Adv Urol. 2010;2(1):19-34.

42. Ruddy KJ, Gelber SI, Tamimi RM, et al. Prospective study of fertility concerns and preservation strategies in young women with breast cancer. J Clin Oncol. 2014;32(11):1151-1156.

43. Cardozo ER, Thomson AP, Karmon AE, Dickinson KA, Wright DL, Sabatini ME. Ovarian stimulation and in-vitro fertilization outcomes of cancer patients undergoing fertility preservation compared to age matched controls: a 17-year experience. J Assist Reprod Genet. 2015; 32(4):587-596.

44. Kim JH, Kim SK, Lee HJ, et al. Efficacy of random-start controlled ovarian stimulation in cancer patients. J Korean Med Sci. 2015; 30(3):290-295

45. Johnson LN, Dillon KE, Sammel MD, et al. Response to ovarian stimulation in patients facing gonadotoxic therapy. Reprod Biomed Online. 2013;26(4):337-344.

46. Pavone ME, Hirshfeld-Cytron J, Lawson AK, Smith K, Kazer R, Klock S. Fertility preservation outcomes may differ by cancer. J Human Reprod Sci. 2014;7(2):111-118

47. Lawrenz B, Fehm T, von Wolff M, et al; Centers of FertiPROTEKT Network. Reduced pretreatment ovarian reserve in premenopausal female patients with Hodgkin lymphoma or non-Hodgkin-lymphoma-evaluation by using antimüllerian hormone and retrieved oocytes. Fertil Steril. 2012;98(1):141-144.

48. Goldman KN, Kramer Y, Hodes-Wertz B, Noyes N, McCaffrey C, Grifo JA. Long-term cryopreservation of human oocytes does not increase embryonic aneuploidy. Fertil Steril. 2015;103(3):662-668.

49. Stoop D, Cobo A, Silber S. Fertility preservation for age-related fertility decline. Lancet. 2014;384(9950):1311-1319.

50. Dolmans MM, Luyckx V, Donnez J, Andersen CY, Greve T. Risk of transferring malignant cells with transplanted frozen-thawed ovarian tissue. Fertil Steril. 2013;99(6):1514-1522.

51. Kyono K, Doshida M, Toya M, Sato Y, Akahira J, Sasano H. Potential indications for ovarian autotransplantation based on the analysis of 5,571 autopsy finding of females under the age of 40 in Japan. Fertil Steril. 2010;93(7):2429-2430.

52. Dolmans MM, Marinescu C, Saussoy P, Van Langendonckt A, Amorim C, Donnez J. Reimplantation of cryopreserved ovarian tissue from patients with acute lymphoblastic leukemia is potentially unsafe. Blood. 2010;116(16):2908-2914.

53. De Vos M, Smitz J, Woodruff TK. Fertility preservation in women with cancer. Lancet. 2014;384(9950):1302-1310. 
54. Dolmans MM, Marotta ML, Pirard C, Donnez J, Donnez O. Ovarian tissue cryopreservation followed by controlled ovarian stimulation and pick-up of mature oocytes does not impair the number or quality of retrieved oocytes. J Ovarian Res. 2014;7:8-84.

55. Prasath EB, Chan ML, Wong WH, et al. First pregnancy and live birth resulting from cryopreserved embryos obtained from in vitro matured oocytes after oophorectomy in an ovarian cancer patient. Hum Reprod. 2014;29(2):276-278.

56. Trudgen K, Ayensu-Coker L. Fertility preservation and reproductive health in the pediatric, adolescent, and young adult female cancer patient. Curr Opin Obstet Gynecol. 2014;26(5):372-380.

57. Gies I, De Schepper J, Tournaye H. Progress and prospects for fertility preservation in prepubertal boys with cancer. Curr Opin Endocrinol Diabetes Obes. 2015;22(3):203-208.

58. Tzakis AG. The first live birth subsequent to uterus transplantation. Transplantation. 2015;99(1):8-9.

59. Bhartiya D, Hinduja I, Patel H, Bhilawadikar R. Making gametes from pluripotent stem cells - a promising role for very small embryonic-like stem cells. Reprod Biol Endocrinol. 2014;12:114-123.

60. Moore HC, Unger JM, Phillips KA, et al; POEMS/S0230 Investigators Goserelin for ovarian protection during breast-cancer adjuvant chemotherapy. N Engl J Med. 2015;372(10):923-932.

61. Cheng TF, Wang JD, Uen WC. Cost-utility analysis of adjuvant goserelin (Zoladex) and adjuvant chemotherapy in premenopausal women with breast cancer. BMC Cancer. 2012;12:33.

62. Peigné M, Decanter C. Serum AMH level as a marker of acute and long-term effects of chemotherapy on the ovarian follicular content: a systematic review. Reprod Biol Endocrinol. 2014;12:26-35.

63. Brougham MF, Crofton PM, Johnson EJ, Evans N, Anderson RA, Wallace WH. Anti-Mullerian hormone is a marker of gonadotoxicity in pre- and postpubertal girls treated for cancer: a prospective study. J Clin Endocrinol Metab. 2012;97(6):2059-2067.

64. Decanter C, Morschhauser F, Pigny P, et al. Anti-Müllerian hormone follow-up in young women treated by chemotherapy for lymphoma: preliminary results. Reprod Biomed Online. 2010;20(2):280-285.

65. Behringer K, Thielen I, Mueller H, et al. Fertility and gonadal function in female survivors after treatment of early unfavorable Hodgkin lymphoma (HL) within the German Hodgkin Study Group HD14 trial. Ann Oncol. 2012;23(7):1818-1825.
66. Lee SJ, Schover LR, Partridge AH, et al. American Society of Clinical Oncology recommendations on fertility preservation in cancer patients. J Clin Oncol. 2006;24(18):2917-2931.

67. Reinecke JD, Kelvin JF, Arvey SR, et al. Implementing a systematic approach to meeting patients' cancer and fertility needs: a review of the Fertile Hope Centers Of Excellence program. J Oncol Pract. 2012; 8(5):303-308.

68. Reyna C, Lee MC. Breast cancer in young women: special considerations in multidisciplinary care. J Multidiscip Healthc. 2014;7:419-429.

69. Shnorhavorian M, Kroon L, Jeffries H, Johnson R. Creating a standardized process to offer the standard of care: continuous process improvement methodology is associated with increased rates of sperm cryopreservation among adolescent and young adult males with cancer. J Pediatr Hematol Oncol. 2012;34(8):e315-e319.

70. Quinn GP, Vadaparampil ST, Gwede CK, et al. Developing a referral system for fertility preservation among patients with newly diagnosed cancer. J Natl Compr Canc Netw. 2011;9(11):1219-1225.

71. Sheth KR, Sharma V, Helfand BT, et al. Improved fertility preservation care for male patients with cancer after establishment of formalized oncofertility program. J Urol. 2012;187(3):979-986.

72. Vadaparampil ST, Hutchins NM, Quinn GP. Reproductive health in the adolescent and young adult cancer patient: an innovative training program for oncology nurses. J Cancer Educ. 2013;28(1):197-208.

73. Murphy D, Orgel E, Termuhlen A, Shannon S, Warren K, Quinn GP. Why healthcare providers should focus on the fertility of AYA cancer survivors: it's not too late! Front Oncol. 2013;3:248-254.

74. Crawshaw M, Sloper PA. Qualitative Study of the Experiences of Teenagers and Young Adults when Faced with Possible or Actual Fertility Impairment Following Cancer Treatment [dissertation]. York: University of York; 2006.

75. Canada AL, Schover LR. The psychosocial impact of interrupted childbearing in long-term female cancer survivors. Psychooncology. 2012;21(2):134-143.

76. Clayman ML, Harper MM, Quinn GP, Reinecke J, Shah S. Oncofertility resources at NCI-designated comprehensive cancer centers. J Natl Compr Canc Netw. 2013;11(12):1504-1509.

77. Geue K, Richter D, Schmidt R, et al. The desire for children and fertility issues among young German cancer survivors. J Adolesc Health. 2014; 54(5):527-535.
Clinical Oncology in Adolescents and Young Adults

\section{Publish your work in this journal}

Clinical Oncology in Adolescents and Young Adults is an international, peer-reviewed, open access journal publishing original research, reports, editorials, reviews and commentaries on all aspects of epidemiology, diagnosis and treatment of cancers in adolescents and young adults. The manuscript management system is completely

\section{Dovepress}

online and includes a very quick and fair peer-review system. Visit http://www.dovepress.com/testimonials.php to read real quotes from published authors. 